\title{
ON THE DEPTH OF BLOWUP ALGEBRAS OF IDEALS WITH ANALYTIC DEVIATION ONE
}

\author{
SANTIAGO ZARZUELA
}

(Communicated by Wolmer V. Vasconcelos)

\begin{abstract}
Let $I$ be an ideal in a local Cohen-Macaulay ring $(A, \mathfrak{m})$. Assume $I$ to be generically a complete intersection of positive height. We compute the depth of the Rees algebra and the form ring of $I$ when the analytic deviation of $I$ equals one and its reduction number is also at most one. The formulas we obtain coincide with the already known formulas for almost complete intersection ideals.
\end{abstract}

\section{INTRODUCTION}

Let $(A, \mathfrak{m})$ be a local ring, and $I$ an ideal of $A$. Denote by ht $(I)$ the height of $I$, and by $l(I)$ the analytic spread of $I$. In [14], S. Huckaba and C. Huneke defined the analytic deviation of $I$ as the diference $l(I)-\mathrm{ht}(I)$. Denote by ad $(I)$ this number. Analytic deviation zero ideals are the so-called equimultiple ideals, which have been extensively studied by several authors (see [7] for an account of the results concerning the Blowing Up rings of these ideals). Recently, many interesting properties about the Rees algebras and the associated graded rings of ideals with small analytic deviation have been discovered, see e.g. $[4,5,6,14,15,20,21,24]$. These results are mainly related to the CohenMacaulay and Gorenstein properties of the Rees algebras and form rings of ideals with analytic deviation 1 or 2 , and they become particularly good when the reduction number of $I$ is also small. Then, there exists a minimal reduction $J$ of $I$ which is very "near" to $I$ and, roughly speaking, one is able to transfer some of the properties of the Rees algebra and the associated ring of $J$ to those of $I$. This point of view has been systematically used by W. V. Vasconcelos in [24] (cf. [22]).

A minimal reduction of a generically complete intersection ideal with analytic deviation 1 is an almost complete intersection (see Section 2 for the definitions of generically complete intersection and almost complete intersection ideals). The Rees algebra and the associated graded ring of almost complete intersection ideals in a Cohen-Macaulay ring are quite well understood, see e.g. [2, 3, 9, 23].

Received by the editors December 9, 1993 and, in revised form, June 23, 1994.

1991 Mathematics Subject Classification. Primary 13A30; Secondary 13C15, 13D45, 13 H10.

Key words and phrases. Rees algebras, form rings, depth, analytic deviation, reduction number.

The author has been partially supported by DGICYT PB 91-0231-C02-01. 
In particular, the depth of both graded rings is known due to M. Brodmann [2] (cf. [9]). Our aim in this note is to obtain similar formulas for the depth of the Rees algebra and the associated graded ring of a generically complete intersection ideal, with analytic deviation 1 and reduction number at most 1 , in a local Cohen-Macaulay ring. This is achieved by means of the following result. Denote by $R(I)$ and $G(I)$, respectively, the Rees algebra and the associated graded ring of $I$, and by $r(I)$ the reduction number of $I$.

Theorem (Theorem 3.1). Let $(A, \mathfrak{m})$ be a local Cohen-Macaulay ring, and $I$ an ideal of $A$ with ht $(I) \geq 1$. Assume I to be generically a complete intersection with ad $(I)=1$. If $r(I) \leq 1$, then the following hold:

(i) depth $G(I)=\min \{\operatorname{dim} A$, depth $A / I+\operatorname{ht}(I)+1\}$.

(ii) depth $R(I)=\min \{\operatorname{dim} A+1$, depth $A / I+\mathrm{ht}(I)+2\}$.

These formulas coincide with the above cited for almost complete intersection ideals.

Now we give a brief description of the content of this note. In Section 2 we collect the most important technical results we shall use in the proof of Theorem 3.1, which is given in Section 3. In Section 4 we give other related results. First, we show that under the conditions of Theorem 3.1, the depth of the Rees algebra of any power of $I$ is the depth of the Rees algebra of $I$, and similarly for the associated graded ring (Theorem 4.3). This result has been proved by J. Ribbe in [19] for almost complete intersection ideals in a Cohen-Macaulay ring. We also show that if $I$ is an ideal which verifies the hypothesis of Theorem 3.1, then the Rees algebra $R(I)$ satisfies Serre's property $S_{2}$, when $A / I$ satisfies $S_{1}$ (Theorem 4.4). The corresponding result for almost complete intersections has been proved by P. Brumatti, A. Simis, and W. V. Vasconcelos in [1].

Throughout this paper, $(A, \mathfrak{m})$ will always be a $d$-dimensional local ring with maximal ideal $\mathrm{m}$ and infinite residue field $A / \mathrm{m}$. If $I$ is an ideal of $A, \mu(I)$ denotes the minimal number of generators of $I$, and $l(I)$ its analytic spread, i.e. the minimal number of generators of a minimal reduction of $I$. The diference $\operatorname{ad}(I)=l(I)-\mathrm{ht}(I)$ is then the analytic deviation of $I$. If $J$ is a minimal reduction of $I$, we shall denote by $r_{J}(I)$ the reduction number of $I$ with respect to $J$, which is the least integer $n$ such that $I^{n+1}=J I^{n}$. The reduction number of $I$ is then defined as $r(I)=\min \left\{r_{J}(I), J\right.$ a minimal reduction of $\left.I\right\}$. The graded ring $R(I)=\bigoplus_{n \geq 0} I^{n} t^{n} \subset A[t]$ is the Rees algebra of $I$, and $G(I)=$ $\bigoplus_{n \geq 0} I^{n} / I^{n+1}$ the associated graded ring of $I$. If $S$ is a Noetherian graded ring defined over $A$ and $\mathscr{M}$ is the maximal homogeneous ideal of $S$, we shall denote by $H_{\mathscr{M}}^{i}(\cdot)$ the $i$-th local cohomology functor with respect to $\mathscr{M}$.

\section{Preliminaries}

Let $I$ be an ideal of $A$. Recall that $I$ is said to be an almost complete intersection if $\mu(I)=\mathrm{ht}(I)+1$, and $I_{\mathfrak{p}}$ is a complete intersection for all minimal prime ideals $\mathfrak{p} \in \operatorname{Min}(A / I)$. Furthermore we say that $I$ is generically a complete intersection if $\mu\left(I_{\mathfrak{p}}\right)=\mathrm{ht}(I)$ for all minimal prime ideals $\mathfrak{p} \in$ $\operatorname{Min}(A / I)$. Our first observation is that a minimal reduction of an analytic deviation one ideal is an almost complete intersection.

Lemma 2.1. Let $(A, \mathfrak{m})$ be a local ring, and $I$ an ideal of $A$. Assume ad $(I)=1$ and $I_{\mathfrak{p}}$ is a complete intersection for all $\mathfrak{p} \in \operatorname{Min}(A / I)$. Let $J$ be a minimal reduction of $I$. Then, $J$ is an almost complete intersection. 
Proof. Since ad $(I)=1, \mu(J)=\mathrm{ht}(I)+1=\mathrm{ht}(J)+1$. Moreover, $\operatorname{Min}(A / I)=$ $\operatorname{Min}(A / J), I_{\mathfrak{p}}$ being a complete intersection for all $\mathfrak{p} \in \operatorname{Min}(A / I)$. Since $J_{\mathfrak{p}}$ is a reduction of $I_{\mathfrak{p}}$, we have $I_{\mathfrak{p}}=J_{\mathfrak{p}}$ for all $\mathfrak{p} \in \operatorname{Min}(A / J)$. Hence $J$ is an almost complete intersection.

If $A$ is Cohen-Macaulay, it is then possible to find nice minimal systems of generators of almost complete intersection ideals, see [8]. The next lemma summarizes the main properties of these families of generators.

Lemma 2.2. Let $(A, \mathfrak{m})$ be a local Cohen-Macaulay ring, and I a generically complete intersection ideal of $A$ with $\mathrm{ht}(I)=g$. Assume $\operatorname{ad}(I)=1$, and let $J$ be a minimal reduction of $I$. Then there exists a family of elements $a_{1}, \ldots, a_{g}, c$ in $J$ such that:

(i) $J_{\mathfrak{p}}=\left(a_{1}, \ldots, a_{g}\right)_{\mathfrak{p}}$ for all $\mathfrak{p} \in \operatorname{Min}(A / I)$.

(ii) $a_{1}, \ldots, a_{g}$ is a regular A-sequence.

(iii) $c$ is a non-zerodivisor of $A$, and $\left(a_{1}, \ldots, a_{g}\right): c=\left(a_{1}, \ldots, a_{g}\right): c^{2}$.

Proof. By Lemma 2.1, $J$ is an almost complete intersection, and $J_{\mathfrak{p}}=I_{\mathfrak{p}}$ for all $\mathfrak{p} \in \operatorname{Min}(A / I)=\operatorname{Min}(A / J)$. Then, apply [8, §4].

The following result of Huckaba and Huneke describes some additional useful properties of these minimal systems of generators.

Lemma 2.3 ([14, Lemma 2.6]). Let $(A, \mathfrak{m})$ be a local Cohen-Macaulay ring, and $I$ an ideal of $A$ with $\mathrm{ht}(I)=g \geq 1$ and $\operatorname{dim} A / I \geq 1$. Assume that $a_{1}, \ldots, a_{g}$ is a regular A-sequence in $I$ that generates $I$ generically. Let $c$ be a non-zerodivisor of $I$ such that $\left(a_{1}, \ldots, a_{g}, c\right) I=I^{2}$. Then:

(i) $\operatorname{depth}\left(A /\left(a_{1}, \ldots, a_{g}\right)^{i}+I^{n}\right) \geq \min \left\{\operatorname{depth} A / I^{i}, \operatorname{dim} A / I-1\right\}$ for all $n \geq 1, i=1, \ldots, n$.

(ii) $\left(a_{1}, \ldots, a_{g}\right)^{i} \cap I^{n}=\left(a_{1}, \ldots, a_{g}\right)^{i} I^{n-i}$ for all $n \geq 1, i=1, \ldots, n-1$.

Now we establish some basic relations between the Rees algebra of an ideal with small analytic deviation and the Rees algebra of its minimal reductions. They can be deduced from several parts of [24]. For completeness we give a proof along with the statement.

Proposition 2.4. Let $(A, \mathfrak{m})$ be a local Cohen-Macaulay ring, and $I$ an ideal of $A$ with $\mathrm{ht}(I)=g \geq 1$. Assume $I_{\mathfrak{p}}$ is a complete intersection for all $\mathfrak{p} \in$ $\operatorname{Min}(A / I)$, and let $J$ be a minimal reduction of $I$. Then:

(i) Suppose $\operatorname{ad}(I)=1$, and let $H_{1}(J)$ be the first Koszul homology with respect to a minimal system of generators of $J$. Then, $H_{1}(J)$ is an A/I-module, and there exists an exact sequence of $A / I$-modules

$$
0 \rightarrow H_{1}(J) \otimes_{A} S \rightarrow A / I \otimes_{A} S \rightarrow R(J) / I R(J) \rightarrow 0 .
$$

(ii) If $r(I) \leq 1, \quad I R(J)=I R(I)$.

Proof. (i) By Lemma 2.1, $J$ is an almost complete intersection. Hence by [9, Proposition (1.6)], Ass $\left(H_{1}(J)\right) \subset \operatorname{Min}(A / J)=\operatorname{Min}(A / I)$. Furthermore, $I_{\mathfrak{p}}=J_{\mathfrak{p}}$ for all $\mathfrak{p} \in \operatorname{Min}(A / I)$. Thus $\left(I H_{1}(J)\right)_{\mathfrak{p}}=0$ for all $\mathfrak{p} \in \operatorname{Min}(A / I)$. Since $\operatorname{Min}\left(I H_{1}(J)\right) \subset$ Ass $\left(H_{1}(J)\right)$, we obtain $I H_{1}(J)=0$. Now set $S$ the polynomial ring $A\left[X_{1}, \ldots, X_{g+1}\right]$, and consider the approximation complex

$$
0 \rightarrow H_{1}(J) \otimes_{A} S \rightarrow A / J \otimes_{A} S \rightarrow G(J) \rightarrow 0,
$$


which is exact since $J$ is an almost complete intersection (see [11, Theorem 4.1]). Tensoring with $A / I$ we have a complex

$$
0 \rightarrow H_{1}(J) \otimes_{A} S \rightarrow A / I \otimes_{A} S \rightarrow R(J) / I R(J) \rightarrow 0 .
$$

Let $L$ be the kernel of the first map. Then $\operatorname{Min}(L) \subset \operatorname{Ass}\left(H_{1}(J) \otimes_{A} S\right)=$ Ass $\left(H_{1}(J)\right) \subset \operatorname{Min}(A / J)=\operatorname{Min}(A / I)$ because $S$ is a free $A$-module. Therefore, the above complex is exact when localized at $\mathfrak{p}$ for all $\mathfrak{p} \in \operatorname{Min}(L)$. Thus $L=0$ and (1) is exact.

(ii) Consider the exact sequence

$$
0 \rightarrow I R(J) \rightarrow I R(I) \rightarrow \bigoplus_{n \geq 0} I^{n+1} / I J^{n} \rightarrow 0
$$

$\left(\bigoplus_{n \geq 0} I^{n+1} / I J^{n}\right.$ is the Sally-module of $I$ with respect to $J$, see [24]). Since $r(I) \leq 1, I^{n+1}=I J^{n}$ for all $n \geq 0$ and the Sally-module vanishes. Thus $I R(J)=I R(I)$.

We finish this section with two variations of [13, Proposition 2.2]. The proof is straightforward, hence we leave it for the reader.

Lemma 2.5. Let $(A, \mathfrak{m})$ be a local ring and $I$ an ideal of $A$.

(i) Assume that $x \in I$ satisfies. $(x) \cap I^{n}=x I^{n-1}$ for all $n \geq 1$. Let $B=A /(x)$ and $I_{1}=I /(x) \subset B$. If $x$ is part of a minimal system of generators of a reduction of $I$, then $l\left(I_{1}\right)=l(I)-1$.

(ii) Let $x \in \mathfrak{m}$ be a non-zero element such that $I^{n}: x=I^{n}$ for all $n \geq 1$. Let $B=A /(x)$ and $I_{1}=I+(x) /(x) \subset B$. Then, $l\left(I_{1}\right)=l(I)$.

\section{Main Result}

Theorem 3.1. Let $(A, \mathfrak{m})$ be a local Cohen-Macaulay ring, and $I$ an ideal of $A$ with $\mathrm{ht}(I) \geq 1$. Assume $I$ to be generically a complete intersection with $\operatorname{ad}(I)=1$. If $r(I) \leq 1$, then the following hold:

(i) depth $G(I)=\min \{\operatorname{dim} A$, depth $A / I+\mathrm{ht}(I)+1\}$.

(ii) depth $R(I)=\min \{\operatorname{dim} A+1$, depth $A / I+\mathrm{ht}(I)+2\}$.

Proof. First of all note that if $\operatorname{depth} A / I \geq \operatorname{dim} A / I-1$, the statement is equivalent to [15, Theorem 2.1], hence we only must prove the case $\operatorname{dim} A / I-$ $2 \geq \operatorname{depth} A / I$. We use induction on depth $A / I$. Assume depth $A / I=0$, and let $J=\left(a_{1}, \ldots, a_{g}, c\right)$ be a minimal reduction of $I$ minimally generated as in Lemma 2.2. Set $S$ equal to the polynomial ring $A\left[X_{1}, \ldots, X_{g+1}\right]$. By Proposition 2.4 (i), we have an exact complex

$$
0 \rightarrow H_{1}(J) \otimes_{A} S \rightarrow A / I \otimes_{A} S \rightarrow R(J) / I R(J) \rightarrow 0,
$$

where $H_{1}(J)$ is the first Koszul homology with respect to $a_{1}, \ldots, a_{g}, c$. By [9, Proposition (1.6)] depth $H_{1}(J) \geq 2$, which implies depth $\left(H_{1}(J) \otimes_{A} S\right) \geq$ $3+$ ht $(I)$. From (1) and by the Depth-Lemma we get depth $(R(J) / I R(J))=$ ht $(I)+1$. Consider now the exact sequence

$$
0 \rightarrow I R(J) \rightarrow R(J) \rightarrow R(J) / I R(J) \rightarrow 0 .
$$

By [9, Proposition 1.7], we have depth $R(J) \geq \mathrm{ht}(J)+2 \geq \mathrm{ht}(I)+2$. Hence from (2) we obtain depth $I R(J)=\mathrm{ht}(I)+2$. On the other hand, $I R(J)=$ $I R(I) \simeq t I R(I)$ by Proposition 2.4 (ii). From the exact sequence

$$
0 \rightarrow t I R(I) \rightarrow R(I) \rightarrow A \rightarrow 0
$$


we then get depth $R(I)=\mathrm{ht}(I)+2$, since $A$ is Cohen-Macaulay. Furthermore, $G(I)$ is not Cohen-Macaulay by [15, Theorem 2.1]. Thus by [16, Corollary 3.12], depth $G(I)=$ depth $R(I)-1=\mathrm{ht}(I)+1$, as we wanted to show.

Now assume depth $A / I>0$. The proof follows by induction on ht $(I)$. Let ht $(I)=1$. Since $\operatorname{dim} A / I \geq 2$, by a similar argument as in the proof of [15, Theorem 2.1], we may find an element $x \in \mathfrak{m}$ such that $x$ is a non-zerodivisor of $A$ and $I^{n}: x=I^{n}$ for all $n \geq 1$. Consider now the ring $B=A /(x)$ and the ideal $I_{1}=I+(x) /(x) \subset B$. Then $\mathrm{ht}\left(I_{1}\right)=1, \operatorname{dim} B / I_{1}=\operatorname{dim} A / I-1$, depth $B / I_{1}=\operatorname{depth} A / I-1, r\left(I_{1}\right) \leq 1$, and $l\left(I_{1}\right)=l(I)$ by Lemma 2.5 . Moreover, $I_{1}$ is generically a complete intersection by the choice of $x$ (see [15, Theorem 2.1]). We then have an exact sequence

$$
0 \rightarrow R(I) \stackrel{\cdot x}{\longrightarrow} R(I) \rightarrow R\left(I_{1}\right) \rightarrow 0 \text {. }
$$

By induction on depth $A / I$ we have

$$
\text { depth } \begin{aligned}
R(I) & =\operatorname{depth} R\left(I_{1}\right)+1=\min \left\{\operatorname{dim} B+1, \mathrm{ht}\left(I_{1}\right)+\operatorname{depth} B / I_{1}+2\right\}+1 \\
& =\min \{\operatorname{dim} A+1, \operatorname{ht}(I)+\operatorname{depth} A / I+2\} .
\end{aligned}
$$

Similarly, from the exact sequence

$$
0 \rightarrow G(I) \stackrel{\cdot x}{\longrightarrow} G(I) \rightarrow G\left(I_{1}\right) \rightarrow 0,
$$

we obtain depth $G(I)=\min \{\operatorname{dim} A, \operatorname{ht}(I)+\operatorname{depth} A / I+1\}$.

If $\mathrm{ht}(I)>1$, let $J=\left(a_{1}, \ldots, a_{g}, c\right)$ be a minimal reduction of $I$ minimally generated as in Lemma 2.2. Following the proof of [15, Theorem 2.1], the initial form $a_{1}^{*}$ of $a_{1}$ is a regular element of degree 1 of $G(I)$. Set $B=A /\left(a_{1}\right)$ and $I_{1}=I /\left(a_{1}\right) \subset B$. By Lemmas 2.3 and 2.5, $l\left(I_{1}\right)=l(I)-1$. Moreover, ht $\left(I_{1}\right)=\mathrm{ht}(I)-1, r\left(I_{1}\right) \leq 1$, and $I_{1}$ is generically a complete intersection. Furthermore, since $a_{1}^{*}$ is regular, we have exact sequences

$$
\begin{gathered}
0 \rightarrow G(I)(-1) \stackrel{a_{1} t}{\longrightarrow} R(I) / a_{1} R(I) \rightarrow R\left(I_{1}\right) \rightarrow 0, \\
0 \rightarrow G(I)(-1) \stackrel{a_{1} t}{\longrightarrow} G(I) \rightarrow G\left(I_{1}\right) \rightarrow 0 .
\end{gathered}
$$

From (7) and by induction, we get

$$
\operatorname{depth} G(I)=\min \{\operatorname{dim} A, \operatorname{ht}(I)+\operatorname{depth} A / I+1\}=\operatorname{depth} R\left(I_{1}\right) .
$$

Thus from (6), depth $R(I)=\min \{\operatorname{dim} A+1, \mathrm{ht}(I)+\operatorname{depth} A / I+2\}$, since $a_{1}$ is a non-zerodivisor of $R(I)$. The proof is now complete.

Example. Let $R=K\left[X_{1}, \ldots, X_{5}\right]$, where $K$ is a field, and $\mathscr{M}=\left(X_{1}, \ldots, X_{5}\right)$. Let $A=R_{\mathscr{M}}$. By [14, Example 4.7], there exists a prime ideal $\mathscr{P}$ in $A$ such that (i) ht $(\mathscr{P})=2$, (ii) $l(\mathscr{P})=3$, (iii) $r(\mathscr{P})=1$, (iv) $\operatorname{depth}(A / \mathscr{P})=1$, and (v) $G(\mathscr{P})$ is not Cohen-Macaulay. By Theorem 3.1, depth $G(\mathscr{P})=4$ and $\operatorname{depth} R(\mathscr{P})=5$.

\section{OTHER RESULTS}

In [19], Ribbe has shown that if $I$ is an almost complete intersection ideal in a Cohen-Macaulay ring $A$, the depth of the Rees algebra of any power of $I$ equals the depth of the Rees algebra of $I$, and similarly for the associated graded rings. Next, we extend these results to analytic deviation one ideals with small reduction number in a Cohen-Macaulay ring. We need the following two lemmas. 
Lemma 4.1. Let $(A, \mathfrak{m})$ be a local ring and $M$ an A-module. Let $r \geq 1$ and set $s=\operatorname{depth} M+r$. Denote by $\mathcal{N}$ the maximal homogeneous ideal of the polynomial ring $A\left[X_{1}, \ldots, X_{r}\right]$. Then, $\left[H_{\mathscr{N}}^{s}\left(M\left[X_{1}, \ldots, X_{r}\right]\right)\right]_{j} \neq 0$ for all $j \leq-r$.

Proof. The proof is by induction on $r$, taking into account for $r=1$ that $M$ is concentrated in degree 0.

Lemma 4.2. Let $(A, \mathfrak{m})$ be a local Cohen-Macaulay ring, and $I$ an ideal of $A$ with ht $(I) \geq 1$. Assume $I$ to be generically a complete intersection with $\operatorname{ad}(I)=1$. Set $s=\min \{\operatorname{dim} A, \operatorname{depth} A / I+\mathrm{ht}(I)+1\}$, and denote by $\mathscr{M}$ the maximal homogeneous ideal of $R(I)$. If $r(I) \leq 1$, then:

(i) $\left[H_{\mathscr{M}}^{s+1}(R(I))\right]_{j} \neq 0$ for all $j \leq-\mathrm{ht}(I)$.

(ii) $\left[H_{\mathscr{M}}^{s}(G(I))\right]_{j} \neq 0$ for all $j<-\mathrm{ht}(I)$.

Proof. Assume first that $\operatorname{depth} A / I \geq \operatorname{dim} A / I-1$. By Theorem 3.1, this implies that both $R(I)$ and $G(I)$ are Cohen-Macaulay. Set $g=h t(I)$. Then $a(G(I))=-g$ by [4, Proposition 2.4], and the statement for $G(I)$ is a consequence of [12, Lemma 4.1]. Consider now the exact sequence

$$
0 \rightarrow I R(I) \rightarrow R(I) \rightarrow G(I) \rightarrow 0,
$$

where depth $G(I)=d$ and depth $R(I)=\operatorname{depth} I R(I)=d+1$. We have monomorphisms $\left[H_{\mathscr{M}}^{d}(G(I))\right]_{j} \hookrightarrow\left[H_{\mathscr{M}}^{d+1}(I R(I))\right]_{j}$, thus $\left[H_{\mathscr{M}}^{d+1}(I R(I))\right]_{j} \neq 0$ for all $j<-g$. On the other hand, from the exact sequence

$$
0 \rightarrow t I R(I) \rightarrow R(I) \rightarrow A \rightarrow 0
$$

we get isomorphisms $\left[H_{\mathscr{M}}^{d+1}(I R(I))\right]_{j-1} \simeq\left[H_{\mathscr{M}}^{d+1}(t I R(I))\right]_{j} \simeq\left[H_{\mathscr{M}}^{d+1}(R(I))\right]_{j}$ for all $j \neq 0$; hence $\left[H_{\mathscr{M}}^{d+1}(R(I))\right]_{j} \neq 0$ for all $j \leq-g$.

Assume now that $\operatorname{depth} A / I \leq \operatorname{dim} A / I-2$. The proof follows by induction on depth $A / I$. If depth $A / I=0$, and using the same notation as in the proof of Theorem 3.1, we have an exact sequence

$$
0 \rightarrow H_{1}(J) \otimes_{A} S \rightarrow A / I \otimes_{A} S \rightarrow R(J) / I R(J) \rightarrow 0,
$$

where $\operatorname{depth}\left(A / I \otimes_{A} S\right)=\operatorname{depth}(R(J) / I R(J))=s$ and $\operatorname{depth}\left(H_{1}(J) \otimes_{A} S\right)>s$. Thus for all $j$ there is an injection of local cohomology modules $\left[H_{\mathscr{N}}^{s}\left(A / I \otimes_{A}\right.\right.$ $S)]_{j} \hookrightarrow\left[H_{\mathcal{N}}^{s}(R(J) / I R(J))\right]_{j}$ and, by Lemma 4.1, $\left[H_{\mathcal{N}}^{s}(R(J) / I R(J))\right]_{j} \neq 0$ for all $j<-g$. Consider now the exact sequence

$$
0 \rightarrow I R(J) \rightarrow R(J) \rightarrow R(J) / I R(J) \rightarrow 0,
$$

where depth $R(J)>s$ and depth $I R(J)=s+1$. We then have injections $\left[H_{\mathcal{N}}^{s}(R(J) / I R(J))\right]_{j} \hookrightarrow\left[H_{\mathscr{L}}^{s+1}(I R(J))\right]_{j}$, where $\mathscr{L}$ is the maximal homogeneous ideal of $R(J)$. Thus $\left[H_{\mathscr{L}}^{s}(I R(J))\right]_{j} \neq 0$ for all $j<-g$. If we consider now the exact sequence

$$
0 \rightarrow t I R(I) \rightarrow R(I) \rightarrow A \rightarrow 0,
$$

we obtain $\left[H_{\mathscr{M}}^{s+1}(R(I))\right]_{j} \simeq\left[H_{\mathscr{M}}^{s+1}(I R(I))\right]_{j-1} \neq 0$ for all $j \leq-g$.

Set $C=\bigoplus_{n \geq 0} I^{n} / J^{n}$. Since $r(I) \leq 1$, there are exact sequences

$$
\begin{aligned}
0 \rightarrow & C(1) \rightarrow G(J) \rightarrow G(J) / I G(J) \rightarrow 0, \\
& 0 \rightarrow G(J) / I G(J) \rightarrow G(I) \rightarrow C \rightarrow 0 .
\end{aligned}
$$


By [9, Proposition (1.7)], we have depth $G(J) \geq s$. From (6) we get depth $C(1)$ $\geq s$; hence from (7) we obtain injections $\left[H_{\mathcal{N}}^{s}(G(J) / I G(J))\right]_{j} \hookrightarrow\left[H_{\mathscr{M}}^{s}(G(I))\right]_{j}$ for all $j$, which implies that $\left[H_{M}^{s}(G(I))\right]_{j} \neq 0$ for all $j<-g$.

Now assume depth $A / I>0$. As in the proof of Theorem 3.1, we follow by induction on $\mathrm{ht}(I)$. Hence if $\mathrm{ht}(I)=1$, we have an exact sequence

$$
0 \rightarrow R(I) \stackrel{\cdot x}{\longrightarrow} R(I) \rightarrow R\left(I_{1}\right) \rightarrow 0,
$$

where depth $R(I)=\operatorname{depth} R\left(I_{1}\right)+1$, ht $\left(I_{1}\right)=$ ht $(I)$, and depth $A / I_{1}=$ depth $A / I$

-1 . Thus for all $j$ we have injections $\left[H_{\mathscr{M}}^{s}\left(R\left(I_{1}\right)\right)\right]_{j} \hookrightarrow\left[H_{\mathscr{M}}^{s+1}(R(I))\right]_{j}$, and by induction $\left[H_{\mathscr{M}}^{s+1}(R(I))\right]_{j} \neq 0$ for all $j \leq-g$. Similarly, from the exact sequence

$$
0 \rightarrow G(I) \stackrel{\cdot x}{\longrightarrow} G(I) \rightarrow G\left(I_{1}\right) \rightarrow 0
$$

we get $\left[H_{M}^{s}(G(I))\right]_{j} \neq 0$ for all $j<-g$.

If ht $(I)>1$ we may construct an ideal $I_{1}$ such that ht $\left(I_{1}\right)=\mathrm{ht}(I)-1$, and exact sequences

$$
\begin{aligned}
0 \rightarrow G(I)(-1) & \stackrel{a_{1} t}{\longrightarrow} R(I) / a_{1} R(I) \rightarrow R\left(I_{1}\right) \rightarrow 0, \\
0 & \rightarrow G(I)(-1) \stackrel{a_{1} t}{\longrightarrow} G(I) \rightarrow G\left(I_{1}\right) \rightarrow 0,
\end{aligned}
$$

where depth $R\left(I_{1}\right)=$ depth $G(I)=s$ and depth $G\left(I_{1}\right)=s-1$. From (11), and by induction, we obtain $\left[H^{s}(G(I))\right]_{j} \neq 0$ for all $j<-g$, and from (10) we obtain $\left[H_{\mathscr{M}}^{s+1}(R(I))\right]_{j} \neq 0$ for all $j \leq-g$, since $a_{1}$ is a non-zerodivisor of $R(I)$. This completes the proof.

Theorem 4.3. Let $(A, \mathfrak{m})$ be a local Cohen-Macaulay ring, and $I$ an ideal of $A$ with $\mathrm{ht}(I) \geq 1$. Assume I to be generically a complete intersection with $\operatorname{ad}(I)=1$. If $r(I) \leq 1$, then for all $n \geq 1$ :

(i) depth $R(I)=$ depth $R\left(I^{n}\right)$.

(ii) depth $G(I)=$ depth $G\left(I^{n}\right)$.

Proof. Statement (i) is clear since $H_{\mathscr{M}}^{i}\left(R\left(I^{n}\right)\right) \simeq H_{\mathscr{M}}^{i}(R(I))^{(n)}$ for all $i$. For (ii) it suffices to apply [19, Lemma (5.3.1)] after Lemma 4.2.

In [1, Proposition (2.6)], Brumatti, Simis, and Vasconcelos proved that if $I$ is an almost complete intersection ideal in a Cohen-Macaulay ring, then $R(I)$ satisfies Serre's condition $S_{2}$. The next result concerns this property for Rees algebras of ideals which satisfy the hypothesis of Theorem 3.1.

Theorem 4.4. Let $(A, \mathfrak{m})$ be a local Cohen-Macaulay ring, and $I$ an ideal of $A$ with $\mathrm{ht}(I) \geq 1$. Assume that $I$ is generically a complete intersection, with $\operatorname{ad}(I)=1$ and $r(I) \leq 1$. If $A / I$ satisfies $S_{1}$, then $R(I)$ satisfies $S_{2}$.

Proof. By [1, Theorem (1.5)], $R(I)$ satisfies $S_{2}$ if and only if $G(I)$ satisfies $S_{1}$ (cf. [17, Theorem 2.2]). Hence it suffices to show that if $\mathscr{P}$ is a homogeneous prime ideal of $G(I)$ with ht $(\mathscr{P}) \geq 1$, then $\mathscr{P} \notin$ Ass $(G(I))$. Let $\mathfrak{p}$ be the inverse image of $\mathscr{P}$ in $A$. By passing to the local ring $A_{\mathfrak{p}}$ we may assume that $\mathfrak{p}=\mathfrak{m}$. Denote by $S$ the polynomial ring $A\left[X_{1}, \ldots, X_{g+1}\right]$, where $g=\mathrm{ht}(I)$, and set $\mathscr{Q}$ as the inverse image of $\mathscr{P}$ in $S$. Consider first the case $\operatorname{dim} A / I \geq$ 
2. Let $J$ be a minimal reduction of $I$ as in Lemma 2.2. By Proposition 2.4

(i) there exists an exact sequence

$$
0 \rightarrow H_{1}(J) \otimes_{A} S \rightarrow A / I \otimes_{A} S \rightarrow R(J) / I R(J) \rightarrow 0 .
$$

By [9, Proposition (1.7)], depth $H_{1}(J) \geq 2$ since $\operatorname{dim} A / J=\operatorname{dim} A / I \geq 2$. Let $\mathscr{T}$ be the ideal $\mathrm{m} S$. Then

$$
\mathscr{T}-\operatorname{depth}\left(A / I \otimes_{A} S\right) \geq 1 \text { and } \mathscr{T}-\operatorname{depth}\left(H_{1}(J) \otimes_{A} S\right) \geq 2 ;
$$

thus from (1) we obtain $\mathscr{T}$ - depth $(G(J) / I G(J)) \geq 1$. Consider the ring $C=\bigoplus_{n \geq 0} I^{n} / J^{n}$. There are exact sequences

$$
\begin{aligned}
0 \rightarrow C(1) & \rightarrow G(J) \rightarrow G(J) / I G(J) \rightarrow 0, \\
0 & \rightarrow G(J) / I G(J) \rightarrow G(I) \rightarrow C \rightarrow 0
\end{aligned}
$$

because $r(I) \leq 1$. By [1, Proposition 2.6], $\mathscr{T}$ - depth $G(J) \geq 1$, hence from (3) we get $\mathscr{T}$ - depth $C(1) \geq 1$. Thus from (2), $\mathscr{T}$ - depth $G(I) \geq 1$, and $\mathscr{Q}$ - depth $G(I) \geq 1$ because $\mathscr{T} \subset \mathscr{Q}$. In particular, $\mathscr{P} \notin \operatorname{Ass}(G(I))$ as we wanted to prove.

Finally, if $\operatorname{dim} A / I \leq 1, G(I)$ is always Cohen-Macaulay by Theorem 3.1. Hence $G(I)$ is $S_{1}$, and the proof is now complete.

Remark 4.5. Note that in Theorem 4.4, the assumption $A / I$ satisfies property $S_{1}$ can be weakened in the following sense: It suffices to assume that if $\operatorname{dim} A / I \geq 2$, then depth $A / I>0$. Recall that if $I$ is an ideal of $A$ with positive grade, the Ratliff-Rush closure of $I$ is defined as $\widetilde{I}=\bigcup_{n \geq 1}\left(I^{n+1}: I^{n}\right)$. In [18], K. Raghavan has shown that if $A$ is a Cohen-Macaulay domain and $I$ is an ideal of $A$ with $\mathrm{ht}(I)=g \geq 1$, which is generically a complete intersection, of analytic deviation 1, and such that all the associated primes of $I$ have height at most $g+1$, and if there exists a minimal reduction $J$ of $I$ such that $r_{J}\left(I_{\mathfrak{p}}\right) \leq 1$ for every prime ideal $\mathfrak{p} \supset I$ with $h t(\mathfrak{p} / I)=1$, then $\tilde{I}=I$. As a consequence of Theorem 4.4 we obtain the following.

Corollary 4.6. Let $(A, \mathfrak{m})$ be a local Cohen-Macaulay ring, and $I$ an ideal of $A$ with $\mathrm{ht}(I)=g \geq 1$. Assume that $I$ is generically a complete intersection with $\operatorname{ad}(I)=1$. Suppose also that the associated primes of $I$ have height at most $g+1$, and that there exists a minimal reduction $J$ of $I$ such that $r_{J}\left(I_{\mathfrak{p}}\right) \leq 1$

for all prime ideals $\mathfrak{p} \supset I$ with $\mathrm{ht}(\mathfrak{p} / I) \leq 1$. Then $\widetilde{I^{n}}=I^{n}$ for all $n \geq 1$.

Proof. By [14, Theorem 2.2] (cf. [24, Theorem 4.7]), we have $r(I) \leq 1$. Thus by Theorem 4.4 we obtain that $R(I)$ is $S_{2}$ (use Remark 4.5), which implies that $\widetilde{I^{n}}=I^{n}$ for all $n \geq 1$, see $[17, \S 2.3]$.

\section{REFERENCES}

1. P. Brumatti, A. Simis, and W. V. Vasconcelos, Normal Rees algebras, J. Algebra 112 (1988), 26-48.

2. M. Brodmann, Rees rings and form rings of almost complete intersections, Nagoya Math. J. 88 (1982), 1-16.

3. S. Goto and Y. Shimoda, On the Gorensteinness of Rees and form rings of almost complete intersections, Nagoya Math. J. 92 (1983), 69-88.

4. S. Goto and S. Huckaba, On graded rings associated to analytic deviation one ideals, Amer. J. Math. 116 (1994), 905-914. 
5. S. Goto and Y. Nakamura, On the Gorensteinness of graded rings associated to ideals of analytic deviation one, Contemp. Math., vol. 159, Amer. Math. Soc., Providence, RI, 1994, pp. 51-72.

6. _ Gorenstein graded rings associated to ideals of analytic deviation two, preprint.

7. M. Herrmann, S. Ikeda, and U. Orbanz, Equimultiplicity and blowing up, Springer-Verlag, New York, 1988.

8. M. Herrmann, B. Moonen, and O. Villamayor, Ideals of linear type and some variants, The Curves Seminar at Queen's, Queen's Papers in Pure and Appl. Math., vol. 6, Queen's Univ., Kingston, Ontario, 1989, p. 83.

9. M. Herrmann, J. Ribbe, and S. Zarzuela, On Rees and form rings of almost complete intersections, Comm. Algebra 21 (1993), 647-664.

10. J. Herzog, A. Simis, and W. V. Vasconcelos, Approximation complexes of blowing up rings, J. Algebra 74 (1982), 466-493.

11. __ Approximation complexes of blowing up rings II, J. Algebra 82 (1983), 53-83.

12. L. T. Hoa and S. Zarzuela, Reduction number and a-invariant of good filtrations, Comm. Algebra 22 (1994), 5635-5656.

13. S. Huckaba, Analytic spread modulo an element and symbolic Rees algebras, J. Algebra 128 (1990), 306-320.

14. S. Huckaba and C. Huneke, Powers of ideals having small analytic deviation, Amer. J. Math. 114 (1992), 367-403.

15. _ Rees algebras of ideals having small analytic deviation, Trans. Amer. Math. Soc. 339 (1993), 373-402.

16. S. Huckaba and T. Marley, Depth formulas for certain graded rings associated to an ideal, Nagoya Math. J. 133 (1994), 57-69.

17. S. Noh and W. V. Vasconcelos, The $S_{2}$-closure of a Rees algebra, Results in Math. 23 (1993), 149-161.

18. K. Raghavan, Powers of ideals generated by quadratic sequences, Trans. Amer. Math. Soc. $\mathbf{3 4 3}$ (1994), 727-747.

19. J. Ribbe, Thesis, Universitat zu Köln, 1991.

20. A. Simis, B. Ulrich, and W. V. Vasconcelos, Cohen-Macaulay Rees algebras and degrees of polynomial relations, Math. Ann. 301 (1995), 421-444.

21. N. V. Trung, Reduction number, a-invariant and Rees algebras of ideals having small analytic deviation, Commutative Algebra (A. Simis, N. V. Trung, and G. Valla, eds.), World Scientific, Singapore, 1984.

22. B. Ulrich, Artin-Nagata properties and reductions of ideals, Contemp. Math., vol. 159, Amer. Math. Soc., Providence, RI, 1994, pp. 373-400.

23. G. Valla, A property of almost complete intersections, Quart. J. Math. Oxford Ser. (2) 33 (1982), 487-492.

24. W. V. Vasconcelos, Hilbert functions, analytic spread, and Koszul homology, Contemp. Math., vol. 159, Amer. Math. Soc., Providence, RI, 1994, pp. 401-422.

Departament d'Algebra i Geometria, Universitat de Barcelona, Gran Via 585, E08007 Barcelona, Spain

E-mail address: zarzuela@cerber.mat.ub.es 